TI 2013-112/I

Tinbergen Institute Discussion Paper

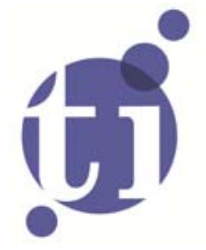

\title{
Superstars Need Social Benefits: An Experiment on Network Formation
}

Boris van Leeuwen

Theo Offerman

Arthur Schram

CREED, Faculty of Economics and Business, University of Amsterdam, and Tinbergen Institute, The Netherlands. 
Tinbergen Institute is the graduate school and research institute in economics of Erasmus University Rotterdam, the University of Amsterdam and VU University Amsterdam.

More TI discussion papers can be downloaded at http://www.tinbergen.nl

Tinbergen Institute has two locations:

Tinbergen Institute Amsterdam

Gustav Mahlerplein 117

1082 MS Amsterdam

The Netherlands

Tel.: +31(0)205251600

Tinbergen Institute Rotterdam

Burg. Oudlaan 50

3062 PA Rotterdam

The Netherlands

Tel.: +31(0)10 4088900

Fax: $+31(0) 104089031$

Duisenberg school of finance is a collaboration of the Dutch financial sector and universities, with the ambition to support innovative research and offer top quality academic education in core areas of finance.

DSF research papers can be downloaded at: http://www.dsf.nl/

Duisenberg school of finance

Gustav Mahlerplein 117

1082 MS Amsterdam

The Netherlands

Tel.: +31(0)20 5258579 


\title{
Superstars Need Social Benefits: An Experiment on Network Formation
}

\author{
Boris van Leeuwen, Theo Offerman and Arthur Schram*
}

19 July 2013

\begin{abstract}
We investigate contributions to the provision of public goods on a network when efficient provision requires the formation of a star network. We provide a theoretical analysis and study behavior is a controlled laboratory experiment. In a $2 \times 2$ design, we examine the effects of group size and the presence of (social) benefits for incoming links. We find that social benefits are highly important. They facilitate convergence to equilibrium networks and enhance the stability and efficiency of the outcome. Moreover, in large groups social benefits encourage the formation of superstars: star networks in which the core contributes more than expected in the stage-game equilibrium. We show that this result is predicted by a repeated game equilibrium.
\end{abstract}

Keywords: Network formation, networked public goods, peer production, social benefits, open source software.

JEL codes: C91, D85, H41

Acknowledgements: We would like to thank Vincent Buskens and Sanjeev Goyal for their helpful suggestions at early stages of the project and audiences at presentations at the Tinbergen Institute, Amsterdam; the CCC Meeting 2012, UEA, Norwich; the International ESA Conference 2012, NYU, New York; the Workshop on Information in Networks (WIN) 2012, NYU Stern, New York; GSU, Atlanta; the IMEBE 2013 conference, Madrid; UPF, Barcelona and KU Leuven for their comments and suggestions. Financial help by the Research Priority Area Behavioral Economics of the University of Amsterdam is gratefully acknowledged.

* CREED, University of Amsterdam and Tinbergen Institute, Roetersstraat 11, 1018 WB Amsterdam, The Netherlands. B.vanLeeuwen@uva.nl, T.J.S.Offerman@uva.nl, Schram@uva.nl. 


\section{$1 \quad$ Introduction}

When individuals acquire and share information, they often do so in groups where they are connected in a network structure. Early work by Lazarsfeld et al. (1944) and Katz and Lazarsfeld (1955) suggests that individuals' roles in the network are distributed in a specific way, where a limited number of individuals influence the majority. This has been observed in applications as diverse as fashion, opinions and voting. Similar findings have been reported more recently. On Wikipedia for instance, a small number of 'Wikipedians' is responsible for the vast majority of articles (Voss, 2005; Ortega et al., 2008). These observations imply that information is typically acquired and shared in networks with a core-periphery structure, where a small core acquires information and a large periphery free-rides.

In this paper, we investigate what drives the formation of such core-periphery networks for the acquisition and sharing of information. An example motivating our research is the development of open source software (OSS). On OSS projects, there are usually a few developers that contribute most of the code while the majority of those involved contribute little or nothing at all (Mockus et al., 2005; von Krogh \& von Hippel, 2006; Crowston et al., 2006). The creation of OSS is a public good: everybody can freely access and change the software code and its use is non-rival. ${ }^{1}$ Hence, there are two ways to access software code: one can either write it personally or use someone else's code.

Galeotti and Goyal (2010) - GG hereafter - show that these patterns may be the result of strategic interaction. In their network formation game, agents desire to access some public good, for instance OSS code, which they can do either by investing personally (writing code) or by making links to others (using someone else's code). Their main result is that in every strict equilibrium of the game, the number of players who invest in the public good is limited. These players - 'the influencers' - form the core of the network. Other (periphery) players link to the core, without contributing themselves. Together, the core and periphery players form a core-periphery network.

The GG model provides a useful theoretical structure for our analysis. In a controlled laboratory experiment, we investigate public good provision in a network environment where players decide both on their contributions to the public good and on their network connections. In particular, we will consider two network characteristics that may affect the structure in which individuals choose to acquire and share information. First, we examine

\footnotetext{
${ }^{1}$ Depending on the specific open source license, there may be license requirements on open source software. For example, it is often required that the updated software is also made freely available (e.g., Fershtman and Gandal 2011).
} 
whether core-periphery networks, or more specifically star networks, form more easily when players in the core receive (social) benefits from incoming links. Second, we investigate the effects of group size on the networks that are formed. In a $2 \times 2$ experimental design, we will systematically vary benefits from incoming links and group size. We vary both in such a way that the (stage-game) equilibrium predictions of the GG model remain unaffected. ${ }^{2}$

Nevertheless, we conjecture that both variations matter. First, if a player derives (social) benefits from incoming links, her payoff is increasing in the number of players that link to her. In contrast, a player's payoff is independent of the number of incoming links without social benefits. In GG's baseline model there are no social benefits. Many applications, though, highlight their role. OSS-developers, for example, may derive positive utility from others using their code. Alternatively, social benefits may represent payments by a third party (Roberts et al., 2006), e.g., through advertisements. The way that we model social benefits allows for either of these interpretations. In the model and in the experiment, the presence of social benefits simply implies that more incoming links will lead to a higher (monetary) payoff.

One feature of the original GG model without social benefits is that at least one player in the core earns a lower payoff than players in the periphery. This is the case, because in equilibrium all players receive the same benefits from the public good, while the costs of investing are higher than the costs of linking. As a result, any player prefers to be in the periphery of the network. This makes it very difficult to coordinate on a core-periphery network. Moreover, it seems counterintuitive that 'the influencers' earn a lower payoff than the periphery players. Introducing benefits for an incoming link can change this payoff asymmetry without changing the equilibrium network structure. With social benefits, the core players may earn more in equilibrium than the periphery players.

Our second variation is in group size. The GG model predicts that the maximum number of players who invest and form the core is independent of group size. We conjecture that group size may matter, especially when there are benefits to incoming links. This is because larger networks have more links and therefore generate more benefits from incoming links. For example, the number of peers that use an OSS developer's code may positively influence her status (Lakhani \& Wolf, 2005; von Krogh \& von Hippel, 2006; Fershtman \& Gandal, 2011) or future job opportunities (Lerner \& Tirole, 2002). Hence, we may see more players in the core when groups are larger. We empirically investigate this conjecture by

\footnotetext{
${ }^{2}$ In section 5 , we show that with repeated interaction social benefits do matter for the equilibrium predictions.
} 
varying the group size between four and eight. Our $2 \times 2$ design allows us to study the interaction between social benefits and group size.

Our results confirm our conjecture that social benefits matter. When they exist, we observe more Nash network architectures, more convergence to stable outcomes and higher levels of efficiency. In the final 10 rounds, we observe Nash architectures (i.e., a coreperiphery structure) in around $75 \%$ of the observations in with social benefits. Without social benefits, groups only form Nash architectures in around $20 \%$ of the observations in the final 10 rounds. Social benefits also lead to more convergence to stable networks. In the two treatments with social benefits, 11 out of the 14 groups converge to a stable network while without benefits only 3 out of the 13 groups converge to a stable network.

The effect of group size is slightly different than conjectured. It turns out that not the number of players in the core is affected, but their investment in the public good. In groups of four and in the presence of social benefits, groups converge mostly to the Nash star network while with groups of eight we observe that groups mostly converge to 'superstars'. In a superstar, the core player invests in more units of the public good than in the Nash star. They do so in a way that makes superstars more efficient than the Nash star network. The formation of superstars is in line with our theoretical result for the finitely repeated game. We show that superstars with a fixed core player can only be supported in a (subgame perfect) repeated game equilibrium if social benefits are present.

We argue that these superstars form because of competition for the core position. With benefits for an incoming link and groups of eight, it becomes very attractive to be in the core of a star. In the first half of the experiment, we observe in most groups that multiple participants compete by investing heavily in the public good after which they converge to a superstar in the second half of the experiment.

We conclude that stars need benefits: the introduction of social benefits has a substantial impact on the rate of convergence. Our main explanation for this effect is that the introduction of social benefits changes the payoff asymmetries between core and periphery players in the network. Superstars need more, however. They require a periphery that consists of sufficiently many players (and the corresponding social benefits) to make heavy investment worthwhile. In all cases, social benefits not only lead to more convergence but also to higher efficiency. With social benefits, payoffs are significantly closer to the socially efficient outcome than without social benefits.

The remainder of this paper is structured as follows. We start with a brief discussion of previous studies in section 2 . We set up the experimental game in section 3 . Section 4 
describes the experimental design and procedures and in section 5 we provide equilibrium and efficiency predictions. The results of the experiment are described in section 6 and section 7 concludes.

\section{Previous Literature}

There is a relatively large theoretical literature on network formation and the provision of public goods in networks, either with endogenously formed networks or exogenously given networks. ${ }^{3}$ Most relevant for our study is the work by Galeotti and Goyal (2010), who extend the network public goods game of Bramoullé and Kranton (2007) by adding endogenous network formation using the protocol designed by Bala and Goyal (2000). As mentioned above, we employ the Galeotti and Goyal (2010) framework in our experiment.

In this brief overview, we focus on experimental work related to ours. Following the boost in network formation theory, the experimental literature on network formation has been steadily growing in recent years. Closest to our study is a recent working paper by Rong and Houser (2012), who investigate network formation and best-shot public good games. Their work differs both in the game that is played on the network and the treatment variables they use. Their game is a best-shot public good game with binary investment: players either invest or not and in equilibrium the (four) players form a star where only the center invests. In their game, the efficient outcome is supported in a Nash equilibrium with a unique network architecture. Hence, efficiency enhancing superstars cannot form in their design. They do not consider the effects of social benefits and group size. In agreement with the results of our treatments without social benefits, they observe Nash networks in only around13 percent of the cases in the baseline treatment. In their design, they vary the action space that is available to the players and find that a restricted action space yields more equilibrium (star) networks.

Within this experimental literature, most relevant here are studies that use the Bala and Goyal (2000) model as the network formation protocol, like we do. A typical result is that groups rarely converge to equilibrium (star) networks (Falk and Kosfeld, 2012). ${ }^{4}$ This holds when players are homogenous in their value to others. With heterogeneity in values,

\footnotetext{
${ }^{3}$ For an overview of the theoretical literature on network formation, see for example Goyal (2007) and Jackson (2008). Other theoretical papers that study public good provision on endogenously formed networks include Cho (2010) and Cabrales et al. (2011). Galeotti et al. (2010), Boncinelli and Pin (2012) and Bramoullé et al. (2012) study public good provision on exogenous networks.

${ }^{4}$ Falk and Kosfeld (2012) do observe equilibrium networks in their treatments with 'one-way flow', i.e. where information only flows in the direction of the player who maintains the link. We study networks with 'two-way flow', i.e. information is exchanged between both players on either side of a link. With this protocol Falk and Kosfeld find hardly any convergence to Nash networks unless they allow for pre-play communication between participants.
} 
star networks form more often (Goeree et al. 2009). The (non-)occurrence of Nash equilibria has mainly been attributed to inequity aversion (Falk and Kosfeld, 2012; Goeree et al., 2009). In environments where the equilibrium payoff differences between core and periphery players are large, Nash networks are typically not observed. The role of inequity aversion is highlighted in Goeree et al. (2009), who estimate the parameters from the Fehr and Schmidt (1999) model and find that subjects experience envy, but no guilt. ${ }^{5}$

Other experimental studies consider public good games with strategic substitutes on a fixed network. Rosenkranz and Weitzel (2012) experimentally investigate the public good game by Bramoullé and Kranton (2007), which is also the game participants play in our endogenous network setting. They vary the (exogenously imposed) network structure between treatments. The results show that it is very difficult for subjects to coordinate in this game, but that on the star and the complete network they find some confirmation of the theory. Charness et al. (2012) test the Galeotti et al. (2010) prediction that when players have incomplete information about the network structure, the multiplicity of equilibria is reduced compared to the case of complete information. Their laboratory results largely support this prediction, as even in the case of incomplete information most groups converge to equilibrium play. ${ }^{6}$

We contribute to this literature in various ways. First of all, we highlight the role of social benefits and payoff asymmetries in network formation and show that the introduction of social benefits increases the rate of convergence and the overall efficiency. Secondly, we show that superstars may form in the (finitely) repeated game equilibrium, but that superstars with a fixed core player can only be part of a repeated game equilibrium with social benefits. Third, we find that superstars are formed with social benefits when group size increases and players jockey to be in the core. Fourth, we show that the core-periphery networks predicted by the GG model are observed in the presence of social benefits. Finally, we show that stable star networks may form when the value of a player is endogenous. This extends the work by Goeree et al. (2009) who show that star networks form under exogenous value-heterogeneity but not with homogeneous values.

\footnotetext{
${ }^{5}$ Berninghaus et al. (2006) also highlight the role of inequity aversion in network formation. In their network formation experiment in continuous time, participants often rotate being the core of the star in order to equalize payoffs. Falk and Kosfeld (2012) also observe rotating, but only if they allow participants to communicate prior to the experiment. We do not observe any behavior consistent with rotating the core position in our data.

${ }^{6}$ There are several other experimental papers that investigate games on an endogenous network (Ule, 2005, Corbae \& Duffy, 2008, Knigge \& Buskens, 2010, Berninghaus et al., 2011, Wang et al., 2012). However, the games they use differ substantially from ours.
} 
Our paper also sheds some light on previous field studies. In a natural field experiment, Zhang and Zhu (2011) investigate contributions to Chinese Wikipedia. They use the repeated blockings of Chinese Wikipedia in mainland China as exogenous variation in group size and observe that contributions increase when groups are larger. Restivo and Van de Rijt (2012) provide a nice example of how social benefits may be operationalized in the field. They show that informal rewards ('barnstars') encourage contributors on Wikipedia to increase their contributions. Our experiment and the GG model jointly provide a possible explanation for both findings. We systematically vary group size and social benefits and show that superstars thrive when they receive social benefits in large groups. We find support for a mechanism where superstars are driven by repeated game effects; from the outset some players contribute a lot to acquire the core position in the star; they continue to perform in an exemplary way until a final phase in which their performance deteriorates.

\section{Experimental game}

In our experiment, we implement a modified version of GG's 'Law of the Few' model. Our main adaptations are that we introduce benefits for an incoming link in some of the treatments and that the investment decision is discrete rather than continuous. ${ }^{7}$ In this section, we will describe the version of the game that we use in the experiment. Section 5 describes the equilibrium and efficiency predictions.

Let $N$ denote the set of players $1,2,3, \ldots, n$. Every player $i \in N$ decides simultaneously on her links $g_{i}$ and her investment $x_{i}$ in some good. Any player $i$ decides on whether to make a link to any of the other players $j \neq i$. If she decides to make a link, we write $g_{i, j}=1$ and $g_{i, j}=0$, if not. By convention, we set $g_{i, i}=0$. The linking decisions of $i$ are summarized by $g_{i}=\left(g_{i, 1}, g_{i, 2}, \ldots, g_{i, n}\right)$ and the linking decisions of all players jointly define the (directed) network architecture $g=\left(g_{1}, g_{2}, \ldots, g_{n}\right)$.

In contrast to the game by GG, investing is a discrete choice. Hence, every player $i \in N$ chooses a positive integer for $x_{i}$, i.e. $x_{i} \in\left\{0,1, \ldots, x_{\max }\right\}$. A strategy of player $i$ is then described by her link and investment decisions, for which we write $s_{i}=\left(g_{i}, x_{i}\right)$, and $i$ 's strategy space is denoted by $S_{i}$. A strategy profile $s$ is the collection of all strategies $s_{i}$ of all $i \in N$. The set of all possible strategy profiles is denoted by $S$.

\footnotetext{
${ }^{7}$ GG also analyze a model allowing for transfers. The main difference with the social benefits in our game is that the size of the transfers in GG equal the costs of making a link, while in our case benefits are strictly smaller than the costs of making a link. Moreover, there is a difference in interpretation; social benefits may be considered to reflect either extrinsic or intrisic motivation, transfers or exogenous income provided by a third party, depending on the application in mind.
} 
Players receive benefits from accessing units of the local public good. The public good is local insofar that any player $i$ accesses her own investment $x_{i}$ and the investments $x_{j}$ of her neighbors $j \in N_{i}$. A player $j$ is a neighbor of $i$, if $i \operatorname{links}$ to $j$ or if $j \operatorname{links}$ to $i$, i.e. $N_{i}=\left\{j \mid \max \left\{g_{i, j}, g_{j, i}\right\}=1\right\}$. The total number of units that $i$ accesses is then given by $y_{i}=x_{i}+\sum_{j \in N_{i}} x_{j}$. The benefits $f\left(y_{i}\right)$ of accessing units are increasing and concave in $y_{i}$. Note that the investment of $i$ and her neighbors are perfect substitutes: $i$ values her own investment the same as any investments by her neighbors.

Investing in units of the good comes at a constant marginal cost of $c_{i}$ per unit. We introduce small heterogeneities in the cost of investment, as we will describe in more detail in the next section. Making a link comes at a cost $k$, which is the same for all players. Linking is less costly than investing, i.e. $c_{i}>k \forall i \in N$. Players receive (social) benefits $b$ from each incoming link. We distinguish between the cases where $b>0$ and $b=0$. Moreover, we take $k>b$, which ensures that making links has a net cost to society. All in all, this results in the following payoff function:

$$
\Pi_{i}(s)=f\left(y_{i}\right)-c_{i} x_{i}-k \sum_{j \in N_{i}} g_{i, j}+b \sum_{j \in N_{i}} g_{j, i}
$$

If we assume self-regarding preferences, a strategy profile $s^{*}$ is a Nash equilibrium if for every player $i \in N$ it holds that

$$
\Pi_{i}\left(s_{i}^{*}, s_{-i}^{*}\right) \geq \Pi_{i}\left(s_{i}, s_{-i}^{*}\right) \forall s_{i} \in S_{i}
$$

where $\Pi_{i}\left(s_{i}^{*}, s_{-i}^{*}\right)$ is the payoff of player $i$ given that she chooses $s_{i}^{*}$ and the other players choose $s_{-i}^{*}$.

\section{$4 \quad$ Experimental design and procedures}

As explained above, we employ a full $2 \times 2$ factorial design that varies the group size and the presence of benefits for an incoming link. Table 1 summarizes this design: we have groups of either 4 or 8 participants, who play the experimental game either with $(b=12)$ or without $(b=0)$ social benefits. 


\begin{tabular}{|c|c|c|c|}
\hline & & \multicolumn{2}{|c|}{ Social benefits } \\
\hline & & $b=0$ & $b=12$ \\
\hline \multirow{2}{*}{ Group size } & $n=4$ & 7 & 8 \\
\hline & $n=8$ & 6 & 6 \\
\hline
\end{tabular}

Previous experimental studies on network formation (Goeree et al., 2009; Falk \& Kosfeld, 2012) have shown that network formation games are rather difficult for experimental participants. Subjects need time to understand the game and to coordinate their actions. To deal with these issues, we implemented a partners design: i.e. participants are randomly assigned a role within a group and play the experimental game for 50 rounds, with fixed partners. ${ }^{8}$ These partners are identified by letters from A to D or A to $\mathrm{H}$, depending on the group size. The number of rounds is announced in the experimental instructions (see Appendix A).

As introducing public good investment makes the game even more difficult than network games with exogenous values, we introduced two additional measures. First, participants are given 10 practice rounds in order to familiarize them with the game. In each of these rounds, participants can try as many decisions as they like during one minute. There is no interaction in these rounds as the decisions of the other players are exogenously given and kept fixed during the practice round. ${ }^{9}$ Second, we introduce small heterogeneities in the costs of investment $c_{i}$ between players. These differences are small enough to keep the theoretical predictions independent of the player: in equilibrium the cost heterogeneities do not determine who will take which position in a network.

In the network game, all participants simultaneously decide on whom to link to and how much to invest. On their decision screen, participants can review all previous decisions in a history box, where their own decisions are marked in orange and the decisions of others in blue. Once everyone in the session has made a decision, participants are informed of the resulting network and their own payoffs. Examples of key screenshots are provided in Appendix B.

\footnotetext{
${ }^{8}$ This corresponds to how OSS developers interact. On many OSS projects, the key contributors remain active over several years (Robles et al., 2005, Crowston et al., 2012)

${ }_{9}^{9}$ The practice rounds can be played online at www.creedexperiment.nl/superstars/extras.php.
} 
TABLE 2: BENEFITS FROM ACCESSING THE PUBLIC GOOD

\begin{tabular}{llllllll}
\hline \hline Units accessed $y_{i}$ & 0 & 1 & 2 & 3 & 4 & 5 & $5+\ell$ \\
Benefits $f\left(y_{i}\right)$ & 0 & 50 & 80 & 90 & 94 & 96 & $96+\ell$ \\
\hline Notes: Benefits from accessing & units of the public good in experimental points.
\end{tabular}

In the experiment, earnings are denoted in 'points'. In addition to the starting capital of 1000 points, subjects earn points in every round. Total point earnings are exchanged at the end of the experiment at a rate of 1 euro for every 140 points. Table 2 gives the benefits function $f(y)$ (in points): the costs of linking $k$ and investing $c_{i}$ are given in Table 3 As specified in section 3 , the function $f(y)$ is increasing and concave in $y$, and $c_{i}>k \forall i$. Note that the cost of investing $c_{i}$ varies by player, where A has the lowest cost of investing and the cost of investing increases by the alphabetical order.

\section{TABLE 3: COSTS OF PUBLIC GOOD INVESTMENT AND LINK FORMATION}

\begin{tabular}{llllllllll}
\hline Role & & A & B & C & D & E & F & G & H \\
Cost per unit of the PG $c_{i}$ & $n=4$ & 23 & 25 & 27 & 29 & & & & \\
Link cost $k$ & $n=8$ & 22 & 23 & 24 & 25 & 26 & 27 & 28 & 29 \\
Notes: Costs per unit of PG investment and costs per link made in experimental points. At the beginning of the \\
experiment, participants are assigned an id, which is denoted by a letter A to H. This id remains constant throughout the \\
experiment.
\end{tabular}

Sessions were run in April 2012 in the CREED-laboratory of the University of Amsterdam and lasted about 2 hours. ${ }^{10}$ In total, 156 subjects participated in the experiment, each in only one session. Participants were recruited from the local CREED database, which consists mostly of undergraduate students from various fields. Of the participants in our experiments, $39 \%$ are female and $60 \%$ were studying at the Faculty of Economics and Business. Cash earnings were between 17.10 euro and 49.50 euro, with a mean of $26.59 .^{11}$

The experiment was computerized using PHP/MySQL and was conducted in Dutch. Upon entering the laboratory, participants were randomly allocated a separate cubicle and communication was prohibited throughout the session. Before starting the network experiment, we elicited risk preferences using a procedure similar to Gneezy and Potters

\footnotetext{
${ }^{10} \mathrm{We}$ also ran pilot sessions based on a strangers design where the participants were provided with a social history screen. We found that coordination is extremely difficult when participants do not have a fixed role and that with fixed roles, stranger rematching, social history screen and social benefits some groups converge to Nash equilibria, but less often than with partner matching.

${ }^{11}$ In one session, participants received an additional 10 euro because the session took almost 3 hours. This is not included in the numbers above. They were not informed about this additional lump-sum payment until the experiment had finished.
} 
(1997). Participants were only informed of the outcome of this part at the very end of the experiment. After this, participants read the instructions of the network game at their own pace, on-screen. While reading the instructions, a printed summary was handed out. To ensure that all participants understood the instructions, they were required to answer several test questions. The experiment would only continue if everyone had answered all questions correctly. The ten practice rounds followed the test questions before the network game started. $^{12}$

We ended each session with a short questionnaire where we gathered some demographic data and asked participants to describe how they had made their decisions in the network game. After this, we privately informed participants of the outcome of the risk elicitation task and their aggregate earnings in the experiment. Participants were privately paid in cash for all rounds of the network game and the risk-elicitation task.

\section{$5 \quad$ Equilibrium and efficiency predictions}

\subsection{Stage-game equilibria}

Here, we derive the stage-game equilibrium predictions for the experimental game described in section 3 and the parameters presented in section 4. If we assume selfish and rational players, the Nash equilibrium networks of the stage game are the same in all experimental treatments, i.e. they are core-periphery networks where either one or two players invest and form the core while the remaining players link with the core and do not invest themselves. ${ }^{13}$ This is also the main result of GG.

Figure 1 and Figure 2 illustrate the Nash equilibrium networks. In these figures, circles represent the players and the numbers inside these circles represent the investment by the player concerned. A link is represented by an arrow, which points away from the player who initiated it. Hence, we see either a Nash star network (Figure 1), where only the single core player invests or a Nash 2-hub network (Figure 2) where both core players invest. In either case, the other players form links to the core and do not invest.

\footnotetext{
${ }^{12}$ An English translation of the handout summary is provided in Appendix A. A translation of the full instructions and the practice questions is available at www.creedexperiment.nl/superstars/extras.php.

${ }^{13}$ The proof follows directly from Proposition 2 in Galeotti and Goyal (2010). Contrary to what is suggested in their Proposition 3, the core player is not uniquely determined in our game even though we have small heterogeneities in the costs of investment. This is because we implement discrete rather than continuous investments.
} 

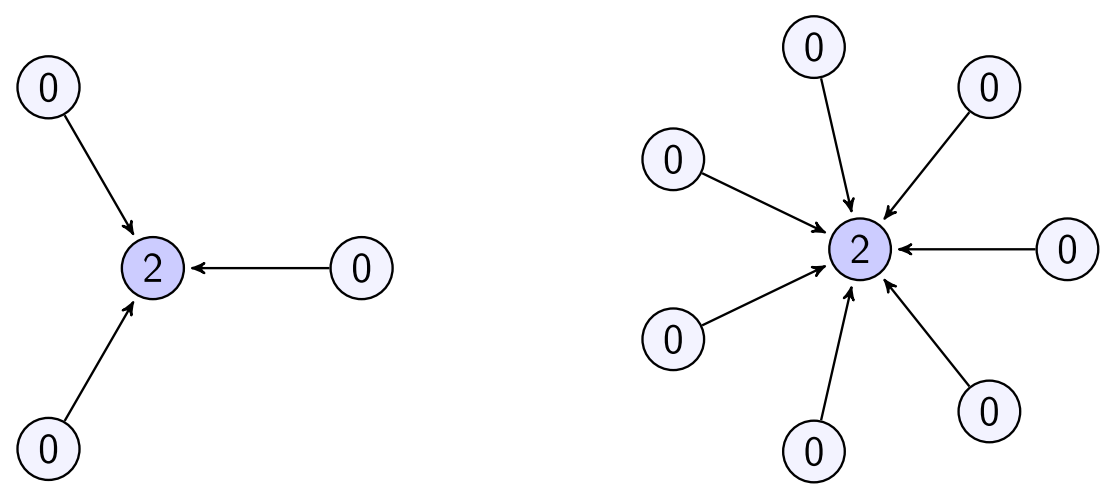

Notes: Nash star networks with $n=4$ and $n=8$ players.

Figure 1 shows Nash star networks with 4 and 8 players. In either case, there is exactly one player who invests in two units of the public good and makes no links while the other players do not invest and make exactly one link to the core-player who invested in the two units. This type of star network is referred to in the literature as a periphery-sponsored star.

\section{FIGURE 2: NASH 2-HUB NETWORKS}
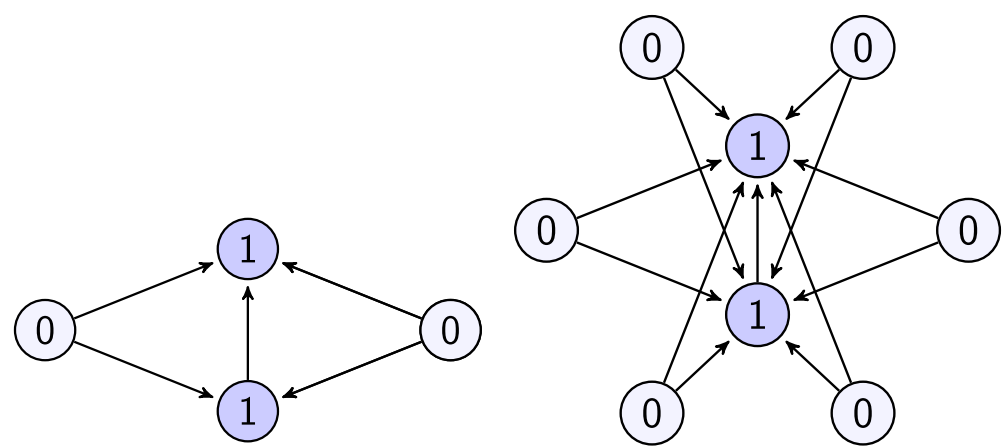

Notes: Nash 2-hub networks with $n=4$ and $n=8$ players.

Figure 2 illustrates Nash core-periphery networks with 2 players in the core, again for groups of 4 and 8 players. We will refer to these networks as Nash 2-hub networks. In these networks, each of the two core players invests in 1 unit and one of the two core players makes a link to the other core player, who makes no links. As in the Nash star, the periphery players do not invest but they do link to (each of) the core players.

The intuition for why these are the possible networks in equilibrium is the following. The marginal benefits of the public good exceed the costs of investing for up to two units of the good. This means that every player wants to access at least two units of the good. Recall that the investments of a player and her neighbors are perfect substitutes. As the costs of making a link are strictly smaller than the costs of investing, i.e. $k<c_{i} \forall i \in N$, any player 
would strictly prefer to access the investment of others rather than investing personally. Combined, this implies that the total investment will sum up to two units. Hence, there will be either one or two players who invest in equilibrium, as is the case in the Nash star and 2hub networks. Note that there are multiple Nash star and Nash 2-hub equilibria, as every player can be in the core of the network in equilibrium, despite the heterogeneities in investment costs. ${ }^{14}$

As mentioned above, the stage-game equilibria are the same in all four experimental treatments. GG show that in equilibrium, the number of players in the core is independent of group size. More specifically, they show that the number of core players $\left|N_{c}\right|$ is bounded from above by $\left|N_{c}\right| \leq \hat{y} \frac{c}{k}$, where $\hat{y}$ is the number of units any player will access in equilibrium. This result carries over to our game, where $\hat{y}=2, c_{i} \in[22,29] \forall i$ and $k=20$; thus $\left|N_{c}\right| \leq 2.9$. Hence, there will be either one or two players in the core of the equilibrium network.

Introducing social benefits does not affect the set of stage-game equilibrium networks. This is the case because, given the strategies $s_{-i}$ of all players $j \neq i$, for any player $i \in N$ a strategy $s_{i}$ yields a (strictly) higher payoff than strategy $s_{i}^{\prime}$ if and only if $\Pi_{i}\left(s_{i}, s_{-i}\right)-$ $\Pi_{i}\left(s_{i}^{\prime}, s_{-i}\right)$ is (strictly) positive. Hence, player $i$ will prefer strategy $s_{i}$ over $s_{i}^{\prime}$ if

$$
\begin{gathered}
f\left(y_{i}\right)-f\left(y_{i}^{\prime}\right)-c_{i}\left(x_{i}-x_{i}^{\prime}\right)-k\left(\sum_{j \in N_{i}} g_{i, j}-\sum_{j \in N_{i}^{\prime}} g^{\prime}{ }_{i, j}\right)+b\left(\sum_{j \in N_{i}} g_{j, i}-\right. \\
\left.\sum_{j \in N_{i}^{\prime}} g_{j, i}\right) \geq 0 .
\end{gathered}
$$

As the linking decisions of all other players are fixed in the above equation, it must be that $\sum_{j \in N_{i}} g_{j, i}=\sum_{j \in N_{i}^{\prime}} g_{j, i}$ and the final term on the left hand side of (3) cancels. Hence, $i$ 's decision is independent of the social benefits level $b$ and the set of equilibrium networks must be independent of $b$.

\footnotetext{
${ }^{14}$ To be precise: there are $n$ different Nash star equilibria and $n(n-1)$ Nash 2-hub equilibria.
} 

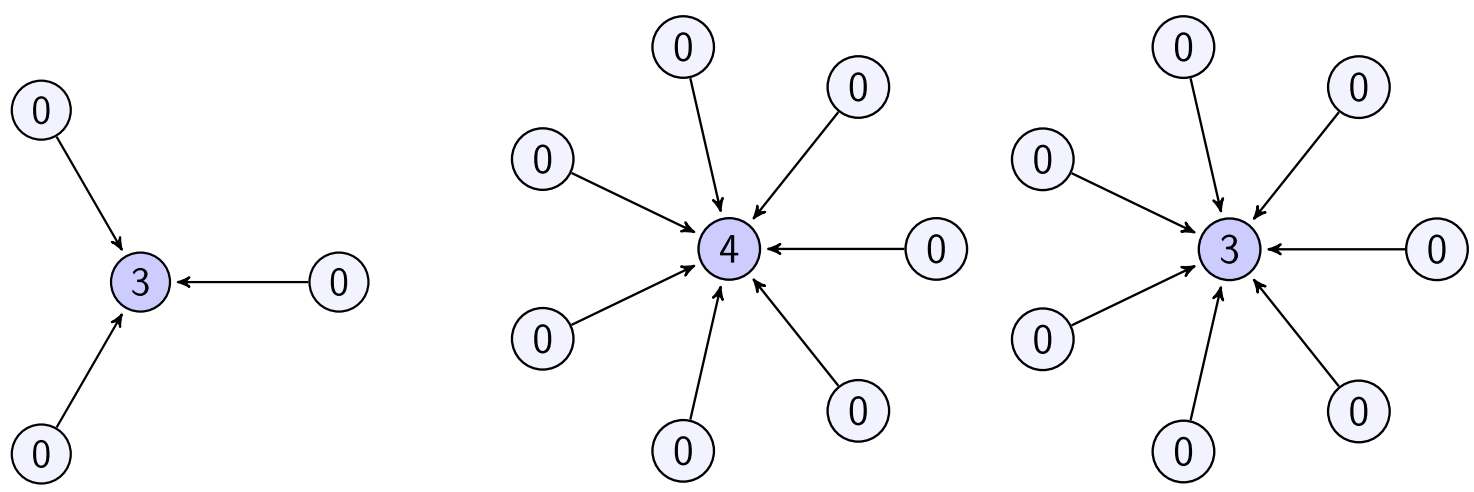

Notes: Examples of superstars. A superstar is a periphery-sponsored star where the core player invests more than in the Nash star. For $n=4$, the superstar where the core player invests in 3 units is an efficient outcome of the game, for $n=8$ this is the superstar where the core-player invests in 4 units.

\subsection{Efficient networks: Superstars}

Next, we consider efficient networks in the distinct treatments. We define social welfare $W$ in a network resulting from a strategy profile $s$ as the sum of all individual payoffs, i.e. $W(s)=\sum_{i \in N} \Pi_{i}(s)$. A network $s$ is called efficient if

$$
W(s) \geq W\left(s^{\prime}\right) \forall s^{\prime} \in S .
$$

Based on this definition, the efficient network is a (minimally-sponsored) star in which the core player invests in more units than in the Nash star and the periphery players do not invest. ${ }^{15}$ If $\mathrm{n}=4$ the core player invests in 3 units and if $\mathrm{n}=8$ she invests in 4 units. $^{16}$

In our analysis, we will also consider superstars. We call a network $s$ a superstar if it is a periphery-sponsored star where the core invests in more units than in the Nash star. Examples of superstars are illustrated in Figure 3. Note that the efficient periphery-sponsored stars are superstars.

\subsection{Repeated game equilibria}

In the experiment, participants play the stage game 50 times in fixed groups. This constitutes a finitely repeated game with a plethora of repeated-game equilibria. In Appendix C, we

\footnotetext{
${ }^{15}$ This follows from Proposition 4 in GG. A minimally-sponsored star is a star network where all connections between the core player and the periphery players are minimally sponsored, i.e. either the core or the periphery player makes a link but not both. Note that the periphery-sponsored star is also a minimally-sponsored star.

${ }^{16}$ Note that in the efficient star networks, the total investment is higher than in the Nash star and that none of the Nash networks is efficient. However, the Nash star is the efficient equilibrium as the sum of all benefits and costs of investment is the same in the Nash star and in the Nash 2-hub, but the sum of link costs and social benefits $\left((k-b) \sum_{i, j \in N} g_{i, j}\right)$ is higher (i.e., more negative) in the Nash 2-hub because there are more links in this network than in a star.
} 
discuss a set of repeated-game equilibria that are more efficient than the repeated play of stage-game equilibria.

GG only provide an analysis for the stage game. Appendix C shows that superstars can be sustained in a (subgame perfect) repeated game equilibrium. For all our treatments, superstars can be sustained until round 48 by rotating the core position. However, we know from previous studies (Goeree et al., 2009; Falk \& Kosfeld, 2012) that coordination is very difficult in network formation experiments. Moreover, in many examples of real world networks, such as OSS projects, the key contributors typically do not change over time (Mockus et al., 2005). If we restrict the set of repeated game equilibria to profiles in which the core position is not rotated, we can show that superstars cannot be sustained without social benefits. In the presence of social benefits however, a superstar with a fixed core can be sustained until the penultimate round.

The intuition behind this result is the following: in the presence of social benefits, the periphery players can induce the core to contribute at the efficient level by the threat that they will remove their links if the core invests too little. This threat has no bite without social benefits.

\section{Results}

In this section, we will present our experimental results. We will provide an overview of the networks we observe, and a discussion of the stability and efficiency of these networks. We will also report the circumstances under which super stars are observed and we will deal with the question of how the emergence of superstars can be explained. Unless stated otherwise, all tests reported in this section are Mann-Whitney tests. Throughout, we use two-sided tests using average statistics per group as units of observation. 


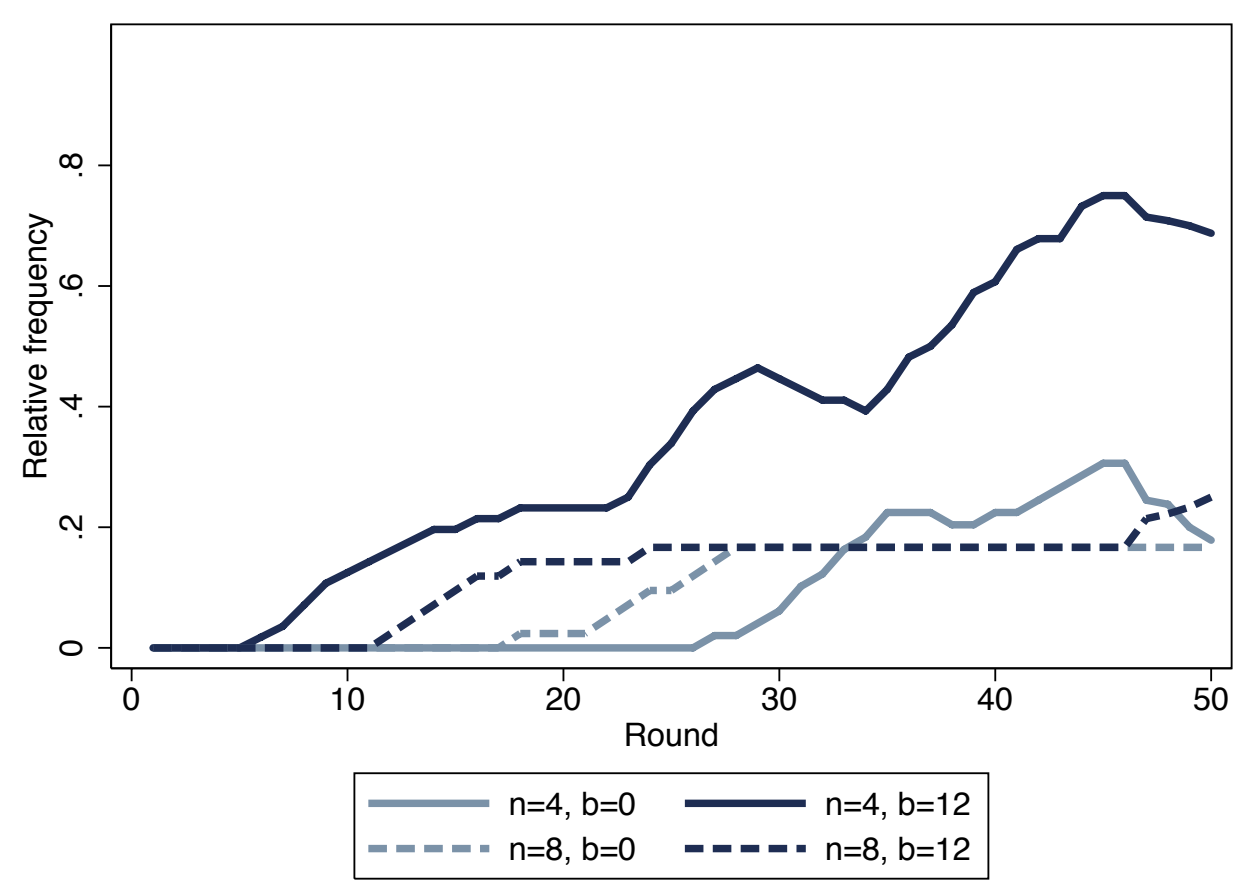

Notes: Lines show the relative frequencies of stage-game equilibria by treatment and round: i.e. all Nash star and Nash 2hub networks. Lines are smoothed by taking the moving average over rounds $t-3$ to $t+3$ for every round $t$.

\subsection{Do groups converge to Nash equilibria?}

First, we consider the question of whether Nash networks are formed. Figure 4 shows the relative frequency of Nash networks over time across treatments. The darker lines refer to the treatments with social benefits and the lighter ones to those without. In addition, the solid lines refer to treatments with groups of 4 while the broken lines refer to treatments with groups of 8 . In the treatment with groups of four and social benefits $(n=4, b=12)$, we find that the relative frequency of Nash networks steadily increases over time. In early rounds we observe few Nash networks, but in the final 10 rounds of this treatment we observe Nash networks in $70 \%$ of the cases.

Nash networks occur considerably less often in the other three treatments. In each of these, the number of Nash networks starts out low and increases over time, but only to a level of around $20 \%$. If we compare the mean number of Nash networks between the social benefit treatments $-(n=4, b=12)$ versus $(n=4, b=0)-$ the difference is significant at the $5 \%$ level, both across all rounds $(p=0.03)$ and for the last 10 rounds $(p=0.04)$. Also when we compare the treatment with social benefits and larger groups $(n=8, b=12)$ to the smaller networks with social benefits $(n=4, b=12)$, we observe significantly fewer Nash networks 
across all rounds $(p=0.04)$ and marginally significantly fewer in the last 10 rounds $(p=0.08) .{ }^{17}$ Other pairwise differences between treatments are statistically insignificant.

\subsection{Do groups converge to Nash architectures?}

It may be difficult for participants to coordinate exactly on Nash networks. Therefore we will first consider Nash architectures, which are all networks where the linking decisions are the same as in a Nash network, but the public good investments may differ. That is, we will look at the occurrence of periphery-sponsored star and periphery-sponsored 2-hub networks, irrespective of investment levels.

Figure 5 plots the relative frequency of Nash architectures over time. Again we see that in treatment $(n=4, b=12)$ the relative frequency steadily increases over rounds, now up to $75 \%$ in the last 10 rounds. This is no surprise, as all Nash networks are also Nash architectures by definition. Interestingly, we now also observe that most groups converge to Nash architectures in the $(n=8, b=12)$ treatment. ${ }^{18}$ In the last 10 rounds we observe Nash architectures in $72 \%$ of the group/round observations in this treatment. For the two treatments without social benefits $(n=4, b=0)$ and $(n=8, b=0)$, we observe Nash architectures in only $31 \%$ and $25 \%$ of the cases, respectively, in the last 10 rounds.

We conclude that groups converge considerably more often to Nash architectures when there are benefits for an incoming link. These differences are also statistically significant. If we compare the treatments with and without social benefits (pooled across group sizes), the difference is significant at the $5 \%$ level, both across all rounds $(p=0.01)$ and the final 10 rounds $(p=0.01)$. If we compare the treatments with and without social benefits for a given group size, the differences are significant for groups of four across all rounds $(p=0.04)$ and marginally so in the last 10 rounds $(p=0.06)$. For groups of eight, the differences are marginally significant in the final 10 rounds $(p=0.09)$, but not across all rounds $(p=0.22)$.

\footnotetext{
${ }^{17}$ A Kruskal-Wallis test comparing all four treatments also rejects the hypothesis that the relative frequency of Nash networks is the same across treatments: $p=0.03$ in all rounds and $p=0.07$ in the last 10 rounds.

${ }^{18}$ The upward trend for the two cases with social benefits is confirmed by statistical testing. Signed Wilcoxon tests reject the hypothesis that the frequency of Nash architectures is the same in the first and second halves of the experiment for the treatments with social benefits $(p=0.02$ for $(n=4, b=12)$ and $p=0.03$ for $(n=8, b=12))$ but not for the treatments without social benefits $(p=0.67$ for $(\mathrm{n}=4, \mathrm{~b}=0)$ and $p=0.49$ for $(\mathrm{n}=8, \mathrm{~b}=0)$.
} 


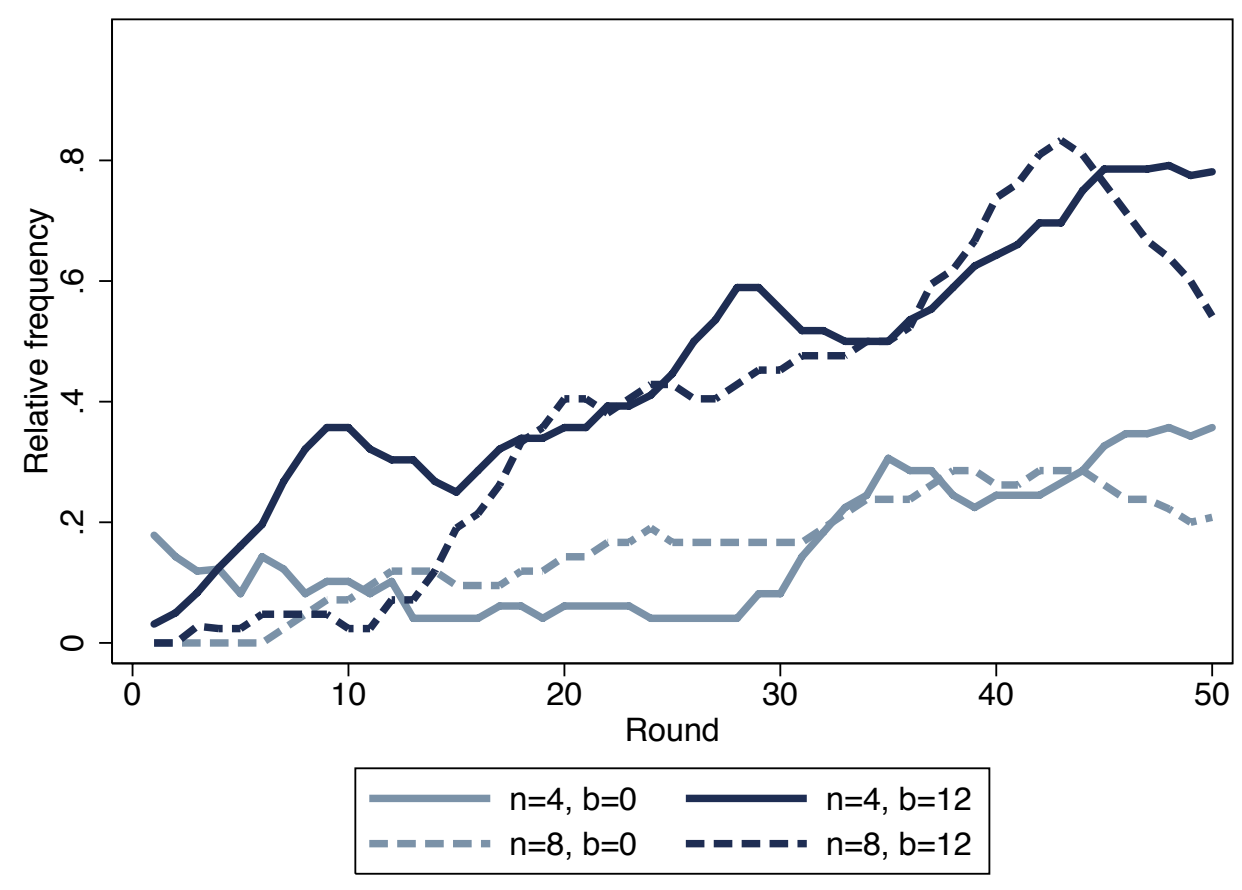

Notes: Lines show the relative frequencies of Nash architectures, i.e. all periphery-sponsored stars and periphery-sponsored 2-hub networks. In a Nash architecture, the network structure is the same as in the Nash networks, but the investments may differ from Nash predictions. Lines are smoothed by taking the moving average over rounds $t-3$ to $t+3$ for every round $t$.

In treatment $(n=8, b=12)$, we observe that most groups play a Nash architecture in the final 20 rounds of the experiment. However, unlike treatment $n=4, b=12$, we do see an end-effect: there is a sharp decline in the number of Nash architectures in the last 10 rounds. We will look at this end-effect in more detail in section 6.4. Before doing so, we will investigate the networks to which groups converge in the different treatments. 


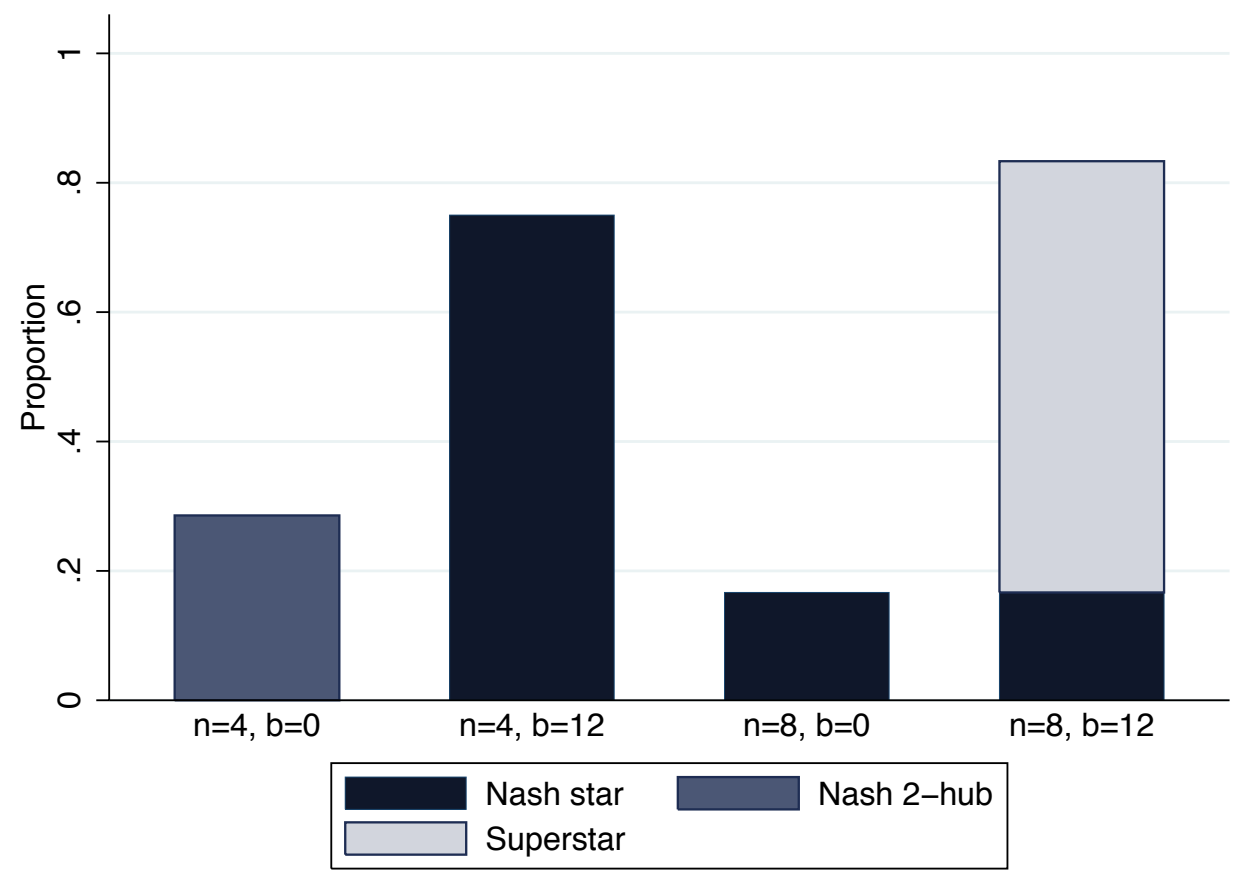

Notes: Bars show the proportion of groups converging by treatment and the network to which a group converges. A group converges to a network if this network is observed for more than 5 consecutive rounds. The graph does not change if we define convergence as observing the same network for either more than 3 or 4 rounds. No group converged to any other network and no group converged to more than one type of network.

\subsection{To which networks do groups converge?}

In the preceding subsections we found that with social benefits, groups converge to Nash architectures most of the time. The question remains, to which networks they converge. Figure 6 shows the proportion of groups that converge to a stable network. A group is said to converge if it is observed to play exactly the same network in more than 5 subsequent rounds, where every player has the same role in each round. ${ }^{19}$ In the two treatments with social benefits, 11 out of 14 groups converge to a stable network, all of which are peripherysponsored stars. Without social benefits, only 3 out of 13 groups converge to a stable outcome. Hence, the introduction of social benefits has a crucial effect on the convergence towards stable networks. The difference in proportions is highly significant (Fisher's exact test, $p=0.01)$.

Aside from the differences in the frequencies of convergence, we observe that groups converge to different networks in distinct treatments. In the treatments with social benefits, groups always converge to periphery-sponsored stars (if they converge). With groups of four this is always the Nash star, while with groups of eight only 1 group converges to the Nash

\footnotetext{
${ }^{19}$ Callander and Plott (2005) and Rosenkranz and Weitzel (2012) use a similar notion of convergence in their analysis.
} 
star, while 4 groups converge to a superstar. Hence, larger groups converge to star networks that are more efficient than the stage-game Nash equilibria. In the treatments without social benefits we observe far less convergence. In $(n=4, b=0)$ we find that only 2 out of 7 groups converge to a stable network and both groups converge to the Nash 2-hub network. In ( $n=4, b=0$ ), only a single group (out of 6 ) converges, this time to the Nash star.

These results that that stars need benefits: we observe considerably more convergence when there are social benefits, both with groups of 4 and groups of 8 players. There is no significant effect of group size on the frequency of convergence (Fisher's exact test, $p=1.00$ ). Group size does affect the level of public good contributions, however, when there are social benefits. With larger groups, the core player invests in more units of the good and superstars form. These superstars are either efficient, or very close to being efficient. We discuss them in more detail in the following subsection.

\subsection{Superstars}

In treatment $(n=8, b=12)$ most groups converge to a superstar: a periphery sponsored star where the core invests in more units than in the Nash star. We rarely observe investment above two units in the other treatments. ${ }^{20}$ The superstars that we observe in treatment ( $n=8, b=12$ ) are efficient or very close to being efficient. Here, we will discuss possible explanations for why superstars are formed in this treatment but not in the other treatments.

In section 5.3 we showed that superstars can be part of a repeated game equilibrium in all treatments. Superstars with a fixed core player however, can only be supported with positive social benefits. This is what we observe in treatment $(n=8, b=12)$ : in the superstars that are formed, the same player always takes the core position. Thus, the observed networks are in line with the theoretical predictions. Note that even though superstars with a fixed core are also part of a repeated game equilibrium in treatment $(n=4, b=12)$, we do not observe stable superstars in this treatment. ${ }^{21}$ An intuitive explanation for this result is that being in the core of the superstar is particularly attractive in large groups. With groups of eight, the core player in a superstar earns a higher payoff than a periphery player in the Nash star, while in groups of four the opposite holds. This makes it worthwhile for periphery

\footnotetext{
${ }^{20}$ More specifically, we observe investment above two units in $1.2 \%$ of the observations in the other treatments.

${ }^{21}$ Two groups did form a superstar in treatment $(n=4, b=12)$, However, one group only stayed in this superstar for two subsequent rounds while the other group only played a superstar in two separate rounds. Of course, we do observe that most groups in this treatment play another repeated game equilibrium: the repeated play of the Nash star.
} 
players in large groups to 'challenge' the core in a Nash star, while such an incentive is lacking in small groups.

Indeed, participants seem to compete for the core position in treatment $(n=8, b=$ 12). ${ }^{22}$ Figure 7 shows the number of players who invest in more than two units of the good in each of the six groups in treatment $(n=8, b=12)$. In all groups we observe some player investing in more than two units of the good, and in five out of six groups we observe multiple participants doing so. In four of these groups, at some point all competitors but one give in and the group converges to a superstar. In one group (group 5), two participants continue to compete for the core position until the final round.

An alternative explanation would be that subjects are concerned about the payoffs of other players; they may for example be inequity averse or altruistic. In the Nash star with eight players, the core player earns about twice as much as the periphery players. She can decrease this payoff difference by investing in more units: all periphery players will benefit while the payoff of the core player drops. To distinguish between this alternative explanation and a repeated game explanation behavior in the final rounds can be used. In the four groups that converge to a superstar, in the final round three core players decrease their investment in the public good to the stage-game Nash level of two, or below. This is illustrated in Figure 7. Groups 1, 2, 3 and 6 converged to a superstar. In the final round, the core players of the superstars in 1, 3 and 6 decreased their investment to two units or less, which they would not have done if they were driven by guilt or altruism. The process of subjects' jockeying for the core position in the early rounds together with the end-round effect support the repeated game interpretation of the results. ${ }^{23}$

\footnotetext{
${ }^{22}$ The benefits function $f(y)$ is such that it is never a best response for a player to link to multiple players if there is one player who invests in two or more units. Hence, the best response for selfish and rational players who do not invest themselves is to make one link to the player with the highest investment.

${ }^{23}$ The beneficial effect of social benefits on coordination on a superstar is reminiscent of a result reported in Brandts and Cooper (2006) and Brandts et al. (2007). In their weakest-link games, increasing the benefits of coordination leads to coordination at higher effort levels. An important difference between their result and ours is that in the weakest link game coordination of the efficient outcome is supported as a Nash equilibrium of the stage game, while we observe that superstars invest more than what is expected in the stage-game equilibrium.
} 

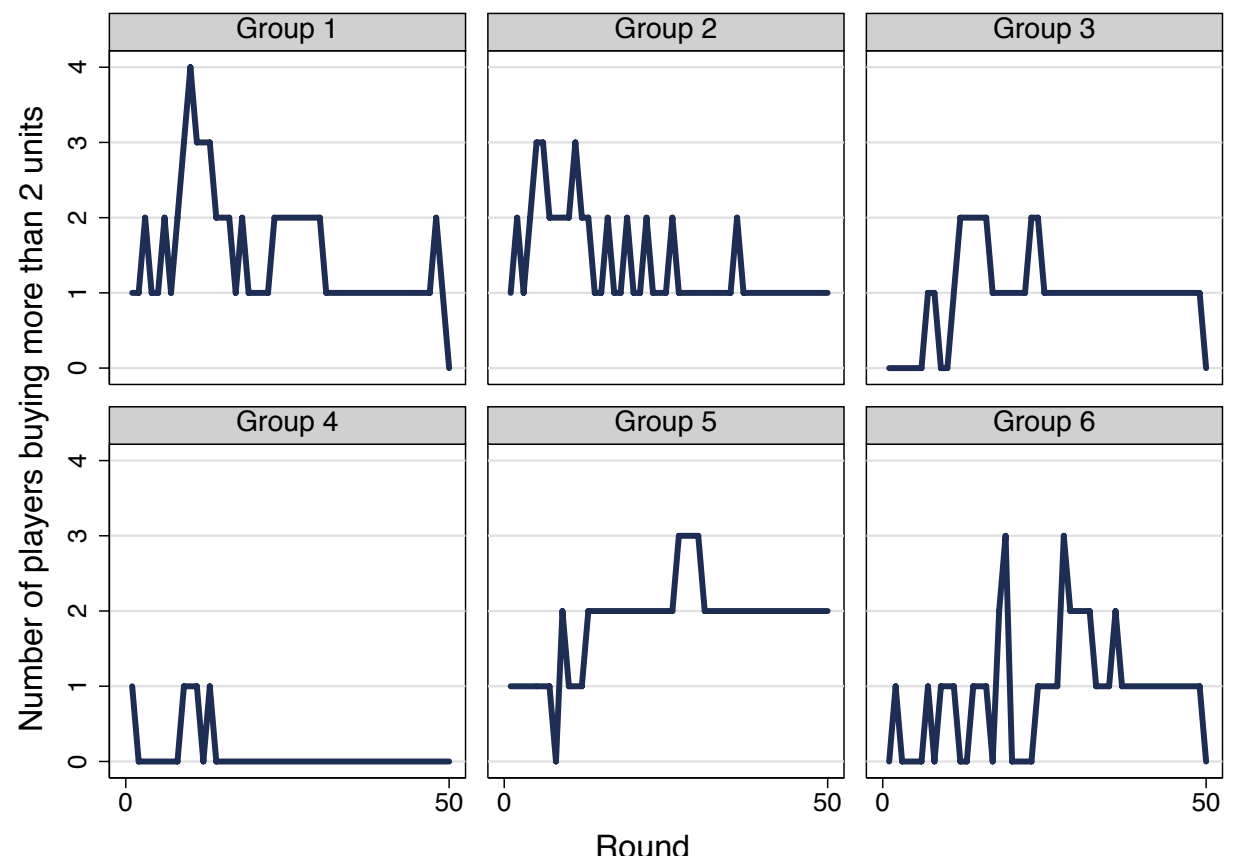

Notes: Lines show the number of participants who buy more than two units of the good in each of the six groups for every round of treatment $(n=8, b=12)$.

\subsection{Frequently observed networks and stability}

If a group converges, it is always to a Nash architecture: we observe groups converging to Nash stars, Nash 2-hubs and superstars. ${ }^{24}$ These networks are also the most frequently observed networks. Table 4 lists the observed frequency of various networks.

Across all treatments, the most frequently observed network is the Nash star. We observe it especially often in treatment $(n=4, b=12)$, where it is formed in 140 of the 400 cases. As described in above, 6 out of 8 groups in this treatment converged to a Nash star and played this network for a substantial number of periods. In treatment $(n=8, b=12)$ we observe a substantial number of superstars: in 75 out of 300 the group/round observations a superstar is formed. Again, in this treatment most groups converged to a superstar.

\footnotetext{
${ }^{24}$ The cost heterogeneities we introduced are by themselves insufficient to achieve coordination: in the treatments without social benefits, we observed convergence in a small number of groups only. If convergence is achieved however (in any treatment), it is indeed usually the low-cost role (A) who acquires the core position. In these treatments, 10 out of 11 groups that converge to a star A in the core, the other group has D in the core. Over all treatments, in $71 \%$ of all stars and in $33 \%$ of all 2-hub networks $\mathrm{A}$ is in the core.
} 
TABLE 4: FREQUENCY OF NASH ARCHITECTURES AND THEIR STABILITY

\begin{tabular}{lcccc}
\hline \hline & \multicolumn{2}{c}{$n=4$} & \multicolumn{2}{c}{$n=8$} \\
& $b=0$ & $b=12$ & $b=0$ & $b=12$ \\
Nash star & $0(-)$ & $140(0.90)$ & $27(0.96)$ & $37(0.97)$ \\
Nash 2-hub & $31(0.77)$ & $1(0.00)$ & $0(-)$ & $0(-)$ \\
Superstar & $0(-)$ & $4(0.00)$ & $0(-)$ & $75(0.76)$ \\
Other star & $19(0.00)$ & $38(0.13)$ & $19(0.05)$ & $3(0.00)$ \\
Other 2-hub & $5(0.00)$ & $1(0.00)$ & $1(0.00)$ & $0(-)$ \\
Other networks & $295(0.01)$ & $216(0.04)$ & $253(0.00)$ & $185(0.04)$ \\
Groups & 7 & 8 & 6 & 6 \\
Observations & 350 & 400 & 300 & 300 \\
: & Cells denote the frequency of the network architectures denoted in the first column in all rounds. The proportion \\
mes that exactly the same network is played in the subsequent round is given between parentheses. The category \\
r stars' refers to periphery-sponsored stars only.
\end{tabular}

Table 4 also lists the stability of the selected networks by treatment. The Nash star is observed to be very stable in all treatments where it is formed: in more than $90 \%$ of the rounds in which it is observed, the Nash star is again formed in the subsequent round. The same is true for the Nash 2-hub network. In treatment $(n=4, b=0)$, we observe the Nash 2hub in 31 rounds and in $77 \%$ of the subsequent rounds the Nash 2-hub is observed again. In treatment ( $n=8, b=12$ ) we frequently observe superstars. They are also very stable: in $76 \%$ of the rounds that a superstar is formed it is also observed in the subsequent round. Note that this is a sizable number: all eight participants are required to make exactly the same decision in the subsequent round. All other networks are observed to be far less stable than Nash networks and superstars. 
TABLE 5: FIRST ROUND OF CONVERGENCE

\begin{tabular}{lclccc}
\hline \hline & & Frequency table & Mean & St. Dev & Groups \\
$n=4$ & $b=0$ & 3243 & 37.50 & 7.78 & 7 \\
& $b=12$ & 92327384144 & 30.33 & 13.26 & 8 \\
$n=8$ & $b=0$ & 25 & 25.00 & - & 6 \\
& $b=12$ & 2127303939 & 31.20 & 7.82 & 6
\end{tabular}

Notes: Cell entries denote the first round from which the same network was played for more than 5 consecutive rounds, i.e. the minimal round t for which the same network is observed in rounds $t$ to $t+5$. The column "Frequency Table" lists this minimal t for each of the groups for which convergence was observed.

Finally, Table 5 indicates how long it takes for groups to converge. Aside from one group that plays the same network from round 9 to 50, groups usually need a substantial number of rounds to converge to a stable network. Most groups need more than 25 rounds to do so. We conclude that in our experiments groups need time to reach a stable network, but once reached they will not easily abandon it.

\subsection{Efficiency analysis}

We now consider treatment differences in observed efficiency. We found that the introduction of social benefits yields more convergence towards Nash architectures. Does this imply that we observe more efficient outcomes? Figure 8 shows the mean relative efficiency per treatment across rounds. We define relative efficiency such that it equals 1 if the efficient outcome is reached and 0 if the sum of payoffs equals the sum of payoffs that would be achieved if there were no interaction, i.e. if all players invest in two units and make no links. $^{25}$

There is a large difference in relative efficiency between treatments with and without social benefits. Across all rounds, the mean relative efficiency of the treatments with social benefits is 0.67 while this is only 0.27 in the treatments without social benefits. This difference is statistically significant, both when we compare groups with- and without social benefits for $n=4(p<0.01)$ and for $n=8(p<0.01)$. Hence, the introduction of social benefits not only leads to more Nash architectures and higher convergence but also to more efficient outcomes.

\footnotetext{
${ }^{25}$ Relative efficiency $r_{t}$ in round $\mathrm{t}$ is defined as $r_{t}\left(s_{t}\right)=\left(W\left(s_{t}\right)-\left(\sum_{i} f(2)-2 c_{i}\right) /\left(W_{\text {max }}-\left(\sum_{i} f(2)-2 c_{i}\right)\right)\right.$, where $W\left(s_{t}\right)$ is the sum of all payoffs in a group in round $t$ and $W_{\max }$ is the maximally attainable sum of payoffs. The Nash star, which is the efficient equilibrium of the stage game, has a relative efficiency of between 0.77 and 0.89 , depending on the treatment.
} 


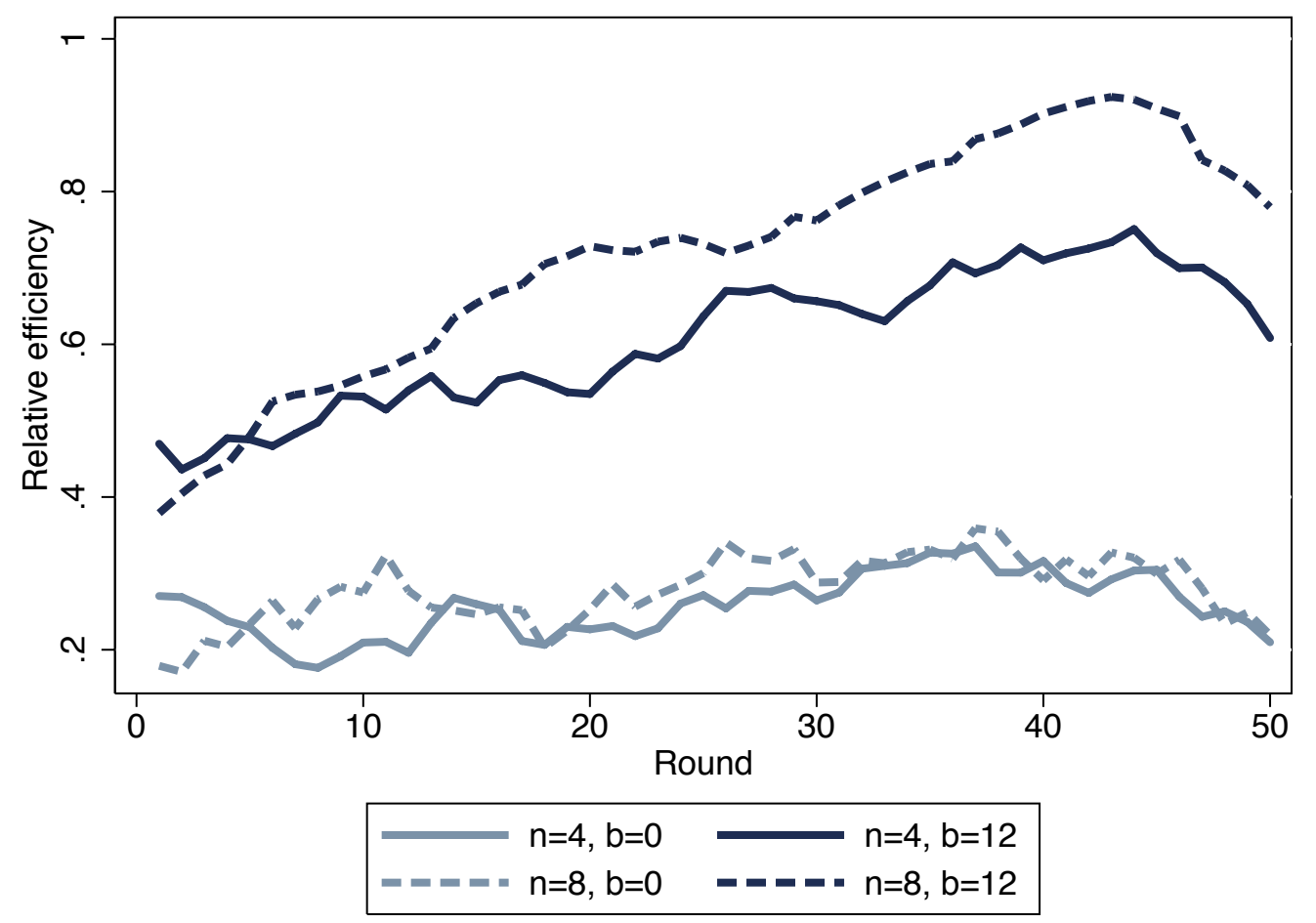

Notes: Lines show mean relative efficiency across rounds by treatment. Relative efficiency in round $t$ is defined as $\left(w\left(s_{t}\right)-\left(\sum_{i} f(2)-2 c_{i}\right) /\left(w_{\max }-\left(\sum_{i} f(2)-2 c_{i}\right)\right)\right.$. Lines are smoothed by taking the moving average over rounds $t-3$ to $t+3$ for every round $t$.

In line with the result that superstars occur frequently in treatment $(n=8, b=12)$ but not in $(n=4, b=12)$, suggests that the relative efficiency is higher in the former treatment. This difference is not statistically significant, however; neither over all rounds $(p=0.20)$ nor in the final 10 rounds $(p=0.24)$.

\section{Conclusion}

We investigated network formation in an environment where players decide both on the network structure and on their contributions to a local public good. For this, we employed the model of Galeotti and Goyal (2010) and systematically investigated the effects of group size and social benefits. We find that the presence of social benefits is crucial for the results. With social benefits, we observe more Nash network architectures, more convergence to stable outcomes and higher levels of efficiency. In combination with a large group size, social benefits lead to the frequent emergence of superstars who contribute the socially efficient level on a Nash network. In stark contrast, groups rarely converge to equilibrium networks, and never to any other stable outcome when there are no social benefits to being in the core of the network. 
A first question one may ask is why we observe convergence to stars with social benefits and not without. This may be related to the ease of coordination. Without benefits, the core player has a lower material payoff than the periphery players. A player finding herself in the core can easily change this situation by no longer investing (but this is not a best response, of course; she cannot make anyone else take over her position). With benefits, the core has higher payoffs than the periphery. Once in that position, a core player has no reason to change.

A related question is why superstars need social benefits? We attribute our results to the repeated nature of the game. Theoretically, superstars can form in any of our treatments as long as players coordinate on a rotation scheme. Such coordination is extremely difficult to accomplish, however. In agreement with previous experimental results and observations outside of the laboratory, our subjects do not rotate the core position once a star network has been formed. If we limit our attention to subgame perfect equilibria where the same player remains in the core of the network, superstars can only form in the presence of social benefits.

In our experimental game the position of the core player in a superstar is only superior in terms of payoffs to the periphery position in the Nash star when groups are large and there are social benefits. In agreement with this observation, we only observe that subjects start by jockeying for the core position in this treatment. Once the dust has settled and subjects have implicitly agreed on who will be the core of the superstar, a stable outcome arises in which the core player consistently contributes more than the stage-game Nash level and all others continue to form links to the core. This convention of playing the game survives until the final phase of the experiment, in which the superstars opportunistically decrease their contributions. Alternatively, one could consider inequity aversion as a reason for superstars to arise in this treatment. We have argued above that this explanation is hard to reconcile with the observation that the core players reduce their investments in the final round of the game.

\section{References}

Bala, V., \& Goyal, S. (2000). A noncooperative model of network formation. Econometrica, 68(5), 1181-1229.

Benoit, J., \& Krishna, V. (1985). Finitely repeated games. Econometrica, 43(4), 905-922.

Berninghaus, S., Ehrhart, K., \& Ott, M. (2006). A network experiment in continuous time: The influence of link costs. Experimental Economics, 9(3), 237-251. 
Berninghaus, S., Schosser, S., \& Vogt, B. (2011). Complexity of networking-an experimental study of the network hawk dove game. Unpublished manuscript.

Boncinelli, L., \& Pin, P. (2012). Stochastic stability in best shot network games. Games and Economic Behavior, 75(2), 538-554.

Bramoullé, Y., Kranton, R., \& D’Amours, M. (2012). Strategic interaction and networks. Unpublished manuscript.

Bramoullé, Y., \& Kranton, R. (2007). Public goods in networks. Journal of Economic Theory, 135(1), 478-494.

Brandts, J., \& Cooper, D. J. (2006). A change would do you good.... an experimental study on how to overcome coordination failure in organizations. American Economic Review, 96(3), 669-693.

Brandts, J., Cooper, D. J., \& Fatas, E. (2007). Leadership and overcoming coordination failure with asymmetric costs. Experimental Economics, 10(3), 269-284.

Cabrales, A., Calvó-Armengol, A., \& Zenou, Y. (2011). Social interactions and spillovers. Games and Economic Behavior, 72(2), 339-360.

Callander, S., \& Plott, C. R. (2005). Principles of network development and evolution: An experimental study. Journal of Public Economics, 89(8), 1469-1495.

Charness, G., Feri, F., Meléndez-Jiménez, M. A., \& Sutter, M. (2012). Equilibrium selection in experimental games on networks. Unpublished manuscript.

Cho, M. (2010). Endogenous formation of networks for local public goods. International Journal of Game Theory, 39(4), 529-562.

Corbae, D., \& Duffy, J. (2008). Experiments with network formation. Games and Economic Behavior, 64(1), 81-120.

Crowston, K., Wei, K., Howison, J., \& Wiggins, A. (2012). Free/libre open-source software development: What we know and what we do not know. ACM Computing Surveys (CSUR), 44(2), 7.

Crowston, K., Wei, K., Li, Q., \& Howison, J. (2006). Core and periphery in free/libre and open source software team communications. Proceedings of the 39th Annual Hawaii International Conference on System Sciences, 2006. HICSS'06. , 6

Falk, A., \& Kosfeld, M. (2012). It's all about connections: Evidence on network formation. Review of Network Economics, 11(3)

Fehr, E., \& Schmidt, K. M. (1999). A theory of fairness, competition, and cooperation*. Quarterly Journal of Economics, 114(3), 817-868. 
Fershtman, C., \& Gandal, N. (2011). A brief survey of the economics of open source software. In S. N. Durlauf, \& L. E. Blume (Eds.), The new palgrave dictionary of economics (Online ed., ) Palgrave Macmillan.

Fudenberg, D., \& Tirole, J. (1991). Game theory MIT Press.

Galeotti, A., Goyal, S., Jackson, M. O., Vega-Redondo, F., \& Yariv, L. (2010). Network games. Review of Economic Studies, 77(1), 218-244.

Galeotti, A., \& Goyal, S. (2010). The law of the few. American Economic Review, 100(4), 1468-1492.

Gneezy, U., \& Potters, J. (1997). An experiment on risk taking and evaluation periods. The Quarterly Journal of Economics, 112(2), 631-645.

Goeree, J. K., Riedl, A., \& Ule, A. (2009). In search of stars: Network formation among heterogeneous agents. Games and Economic Behavior, 67(2), 445-466.

Goyal, S. (2007). Connections: An introduction to the economics of networks Princeton University Press.

Jackson, M. O. (2008). Social and economic networks Princeton University Press.

Katz, E., \& Lazarsfeld, P. F. (1955). Personal influence : The part played by people in the flow of mass communications. Glencoe, Ill.: Free Press.

Knigge, A., \& Buskens, V. (2010). Coordination and cooperation problems in network good production. Games, 1(4), 357-380.

Lakhani, K. R., \& Wolf, R. G. (2005). Why hackers do what they do: Understanding motivation and effort in free/open source software projects. In J. Feller, B. Fitzgerald, S. A. Hissam \& K. R. Lakhani (Eds.), Perspectives on free and open source software (pp. 3-21) MIT Press.

Lazarsfeld, P. F., Berelson, B. R., \& Gaudet, H. (1944). The people's choice : How the voter makes up his mind in a presidential campaign. New York: Columbia University Press.

Lerner, J., \& Tirole, J. (2002). Some simple economics of open source. The Journal of Industrial Economics, 50(2), 197-234.

Mockus, A., Fielding, R. T., \& Herbsleb, J. D. (2005). Two case studies of open source software development: Apache and mozilla. In J. Feller, B. Fitzgerald, S. A. Hissam \& K. R. Lakhani (Eds.), Perspectives on free and open source software (pp. 163-209) MIT Press.

Ortega, F., Gonzalez-Barahona, J. M., \& Robles, G. (2008). On the inequality of contributions to wikipedia. Hawaii International Conference on System Sciences, Proceedings of the 41st Annual, 304-304. 
Restivo, M., \& van de Rijt, A. (2012). Experimental study of informal rewards in peer production. PloS One, 7(3), e34358.

Roberts, J. A., Hann, I. H., \& Slaughter, S. A. (2006). Understanding the motivations, participation, and performance of open source software developers: A longitudinal study of the apache projects. Management Science, 52(7), 984-999.

Robles, G., Gonzalez-Barahona, J. M., \& Michlmayr, M. (2005). Evolution of volunteer participation in libre software projects: Evidence from debian. Proceedings of the 1st International Conference on Open Source Systems, 100-107.

Rong, R., \& Houser, D. (2012). Growing stars: A laboratory experiment on network formation. Unpublished manuscript.

Rosenkranz, S., \& Weitzel, U. (2012). Network structure and strategic investments: An experimental analysis. Games and Economic Behavior, 75(2), 898-920.

Ule, A. (2005). Exclusion and cooperation in networks. (PhD thesis, Tinbergen Institute and Universiteit van Amsterdam).

von Krogh, G., \& von Hippel, E. (2006). The promise of research on open source software. Management Science, 52(7), 975-983.

Voss, J. (2005). Measuring wikipedia. International Conference of the International Society for Scientometrics and Informetrics, 221-231.

Wang, J., Suri, S., \& Watts, D. J. (2012). Cooperation and assortativity with dynamic partner updating. Proceedings of the National Academy of Sciences, 109(36), 14363-14368.

Zhang, X., \& Zhu, F. (2011). Group size and incentives to contribute: A natural experiment at chinese wikipedia. American Economic Review, 101(4), 1601-1615. 


\section{Appendix A: $\quad$ Summary of the Instructions}

Below is translation of the summary of the (Dutch) instructions, which was handed out on paper to the participants. All treatment dependent text is marked in italics. Full instructions for each of the treatments are available on www.creedexperiment.nl/superstars/extras.php.

\section{Summary of the instructions}

You can earn points during the experiment. These points are worth money. How many points (and hence how much money) you earn, depends on your own decisions, the decisions of others and luck. At the end of the experiment your earned points will be converted to euros and the earned amount will be paid to you in private. Your total earnings consist of the points you earn in the first part of the experiment (the lottery) and the sum of all points that you earn in the second part of the experiment. At the beginning of the second part you will receive a starting capital of 1000 points. This will also be added to your earnings.

\section{Every 140 points are equivalent to 1 euro.}

The second part of the experiment consists of 50 rounds. At the beginning of the experiment, you will randomly placed in a group of 8 participants. The composition of this group will not change during the experiment. In this group you will be randomly assigned a role. This role will be indicated by a letter: “ $A$ ”, “ $B$ ”, “ $C$ ”, “ $D$ ”, “ $E$ ”, “ $F$ ”, “ $G$ ” or " $H$ ”. The letters " $A$ ", " $B$ ”, “ $C$ ”, " $D$ ”, " $E$ ", “ $F$ ", " $G$ ” and " $H$ " will thus refer to the same participant throughout the entire experiment.

Every round you can earn points by having 'access' to units of a good. The number of points that you earn depends on the number of units that you have access to. This is shown in the following table:

\begin{tabular}{|c|c|c|c|c|c|c|c|c|c|c|c|c|}
\hline Units & 0 & 1 & 2 & 3 & 4 & 5 & 6 & 7 & 8 & 9 & 10 & $10+i$ \\
\hline Benefits & 0 & 50 & 80 & 90 & 94 & 96 & 97 & 98 & 99 & 100 & 101 & $101+i$ \\
\hline
\end{tabular}

These benefits are the same for each role and all participants in this experiment. The table shows for instance that you earn 80 points if you have access to 2 units and that you earn 100 points if you have access to 9 units of the good. 
There are three ways to access units of the good.

1. You buy units of the good yourself.

2. You make a 'link' to another participant. In this case you have access to the units that the other participant has bought and the other participant has access to your purchases. How you make a link will be explained in a moment.

3. Another participant makes a link to you. In this case you have access to the units that the other participant has bought and the other participant has access to your purchases.

If you make a link to another participant and this other participant also makes a link to you, you will only access the entire purchase of the other participant once.

In addition, you can earn points if other participants make a link to you. For each link that another participant makes to you, you will receive 12 points.

Buying units and making links is costly. The cost of making a link is the same for all roles but the costs of buying units differ by role.

The cost of making a link is 20 points for each role. The cost of making a link are only paid by the participant who makes the link. Every round, you can maximally make one link to each of the other roles. This means that you can maximally make 7 links.

The costs of buying units differ by role. The costs of buying units of the good are given in the table below. Every round, you can maximally buy 5 units of the good.

\begin{tabular}{|l|l|l|l|l|l|l|l|l|}
\hline Role & $A$ & $B$ & $C$ & $D$ & $E$ & $F$ & $G$ & $H$ \\
\hline Cost per unit & 22 & 23 & 24 & 25 & 26 & 27 & 28 & 29 \\
\hline Cost per link & 20 & 20 & 20 & 20 & 20 & 20 & 20 & 20 \\
\hline Benefits per link to your role & 12 & 12 & 12 & 12 & 12 & 12 & 12 & 12 \\
\hline
\end{tabular}




\section{Appendix B: $\quad$ Screen shots}

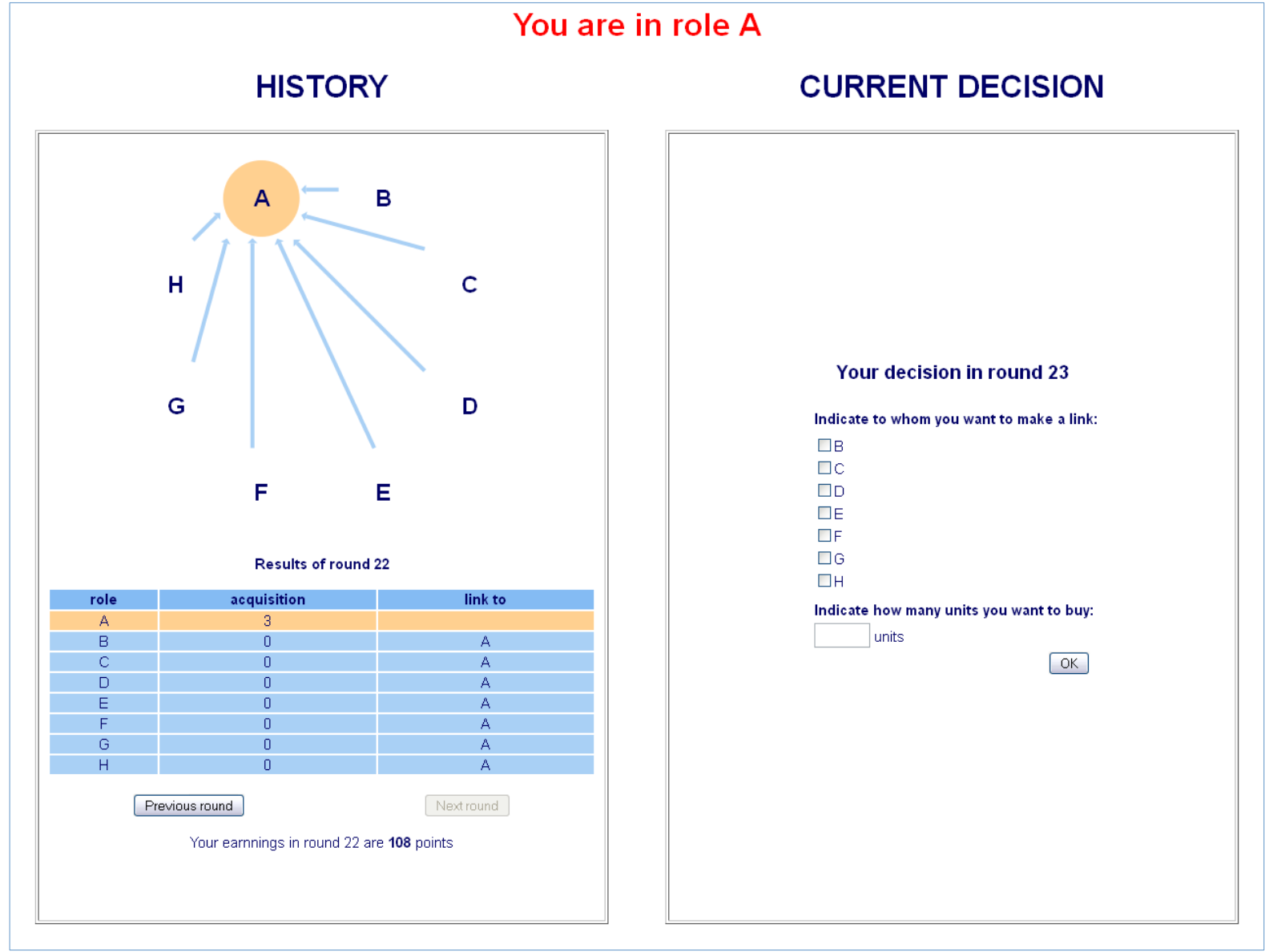

\section{RESULTS}

A

B

C

D

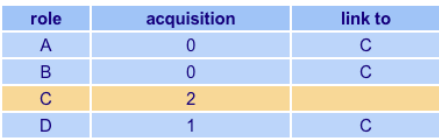

Round 12 of 50 is over

You have made no links and $A, B$ and $D$ made $a$ link to you.

This means that you have access to the acquisitions of $\mathrm{A}, \mathrm{B}$ and $\mathrm{D}$ and your own acquisition.

In total you have access to 3 units, your benefits of this are 90 points.

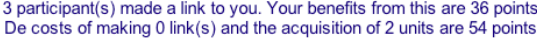

Toure total earnings are 72 points in this round.

You have earned 3231 points in total. 


\section{Appendix C: Repeated game equilibria}

In this Appendix we analyze the finitely repeated game. As usual, this game has a plethora of repeated game equilibria, including repetition of the stage-game equilibrium in each of the T periods. Here, we are interested in equilibria in which players earn higher payoffs than when they repeatedly play a stage-game equilibrium. We focus on equilibria in which an efficient network is consistently formed. In addition, we restrict our attention to strict subgame perfect equilibria. In the GG model, efficient networks are all superstars. We denote the efficient investment level by the core player in the superstar by $\tilde{y}$.

One way to support efficient equilibria is by the use of punishment strategies. Like Benoit and Krishna (1985) we consider the use of 'optimal punishments'. The optimal punishment strategy yields the worst possible payoff for the punished player $i$ that is feasible in a subgame perfect equilibrium. The average payoff for the punished player $i$ from the optimal punishment in $K$ periods of punishment is denoted by $w_{i}(K) / K$, where $w_{i}(K)$ is the total payoff of the punished player in these $K$ periods. Benoit and Krishna show that this payoff is bounded by:

$$
v_{i} \leq w_{i}(K) / K \leq w_{i}(1)
$$

where $v_{i}$ is $i$ 's minmax payoff and $w_{i}(1)$ her worst possible payoff in a stage-game equilibrium.

Proposition 1: In any of our experimental games, superstars can be supported as part of a subgame perfect equilibrium until period $T-2$.

Proof. One way to support superstars is by rotating the core position. Suppose that all players observe a perfectly correlated signal $\theta_{t}$ at the beginning of each period $t$. This signal is an independent draw from the set $\{1, \ldots, n\}$. Each integer is drawn with equal probability. ${ }^{26}$ Consider the strategy profile $s^{*}$, where each player's strategy $s_{i}^{*}$ adheres to:

\footnotetext{
${ }^{26}$ Superstars can also be supported until period $T-2$ if the core position is rotated deterministically, i.e. if there is a sequence $\{1,2, \ldots, n-1, n, 1,2, \ldots\}_{t=1}^{t=T}$ that determines which player will be in the core. This proof is more involved however. For expositional reasons, we chose to include the 'sunspot' equilibrium as our main point is that by rotating, superstars can be part of a repeated game equilibrium in each of our treatments.
} 
"In each period, the player to fill the core position is determined by the draw of $\theta_{t}$, i.e. if $\theta_{t}=j$, player $j$ will be in the core position in period $t$. In a period, the core player does not form any links, and all other players only form a link to this core player. In the first $T-Q$ periods the core player invests the efficient level $\tilde{y}$ and in the final $Q$ periods the core player invests the stage-game Nash level $\hat{y}$. If some player $i$ deviates in round $t$, play switches to the punishment regime and the deviating player will be punished in rounds $t+1$ to $T$ by the optimal punishment strategy."

The strategy profile $s^{*}$ constitutes a subgame perfect equilibrium if it satisfies the one-stagedeviation principle. That is, the strategy profile $s^{*}$ is a subgame perfect equilibrium if and only if :

$$
\pi_{i}\left(s_{i}^{*}, s_{-i}^{*}\right)>\pi_{i}\left(s_{i}^{\prime}, s_{-i}^{*}\right) \forall i \in N, \forall s_{i}^{\prime},
$$

where $s_{i}^{\prime}$ indicates a deviant strategy of player $i$, which differs from the equilibrium strategy $s^{*}$ only in period $t$ and conforms to $s^{*}$ thereafter (see for instance, Theorem 4.1 in Fudenberg and Tirole (1991, p.109) for a proof).

Let $\pi_{C}^{S}$ and $\pi_{P}^{S}$ denote the stage-game payoff of being, respectively, in the core or the periphery of the efficient superstar. Likewise, we write $\pi_{C}^{N}$ and $\pi_{P}^{N}$ for the payoff of being, respectively, in the core or the periphery of the Nash star.

In $s^{*}$, the final $Q$ periods consist of a sequence of stage-game equilibria, hence, a profitable one-stage-deviation can only exist in the first $T-Q$ periods. First consider deviations by the core in these periods. The optimal deviation by the core player in a superstar is to invest in the Nash level $\hat{y}$ which implies that a Nash star would result. Hence, by deviating in period $t \leq T-Q$, the sum of payoffs in periods $t$ to $T$ of core player $i$ will be:

$$
\pi_{i}\left(s_{i}^{\prime}, S_{-i}^{*}\right)=\pi_{C}^{N}+(T-t) \frac{w_{i}(T-t)}{T-t} .
$$

The expected payoff from following $s_{i}^{*}$ is $\bar{\pi}^{S}=\frac{n-1}{n} \pi_{P}^{S}+\frac{1}{n} \pi_{C}^{S}$ in each of the first $T-Q$ periods and $\bar{\pi}^{N}=\frac{n-1}{n} \pi_{P}^{N}+\frac{1}{n} \pi_{C}^{N}$ in each of the final $Q$ periods. In every period $t \leq T-Q$, the core player in the superstar will not deviate if: 


$$
\pi_{C}^{S}+(T-Q-t) \bar{\pi}^{S}+Q \bar{\pi}^{N}>\pi_{C}^{N}+(T-t) \frac{w_{i}(T-t)}{T-t} .
$$

As $\bar{\pi}^{S}>\bar{\pi}^{N}>w_{i}(1) \geq \frac{w_{i}(T-t)}{T-t}$, it suffices to consider only period $t=T-Q$. Using this, we can rewrite (C.4) to:

$$
Q>\frac{\pi_{C}^{N}-\pi_{C}^{S}}{\bar{\pi}^{N}-w_{i}(Q) / Q}
$$

which gives a condition for the minimal length of the 'Nash phase' that is needed to avoid deviation by the core. As $\bar{\pi}^{N}>w_{i}(1) \geq \frac{w_{i}(T-t)}{T-t}$ and $\pi_{C}^{N}>\pi_{C}^{S}$, the right hand side of (C.5) is always positive.

If $b=0$, we have $v_{i}=w_{i}(1)=\pi_{C}^{N}$. Using this in (C.5), we can compute for our game that $Q \geq 1$ if $n=4, b=0$ and $Q \geq 2$ if $n=8, b=0 .{ }^{27}$ Similarly, in our games with social benefits, the worst stage-game equilibrium payoff is the periphery position in the Nash 2-hub. Using this as the average punishment payoff $w_{i}(Q) / Q$ in case of deviation, we find that $Q \geq 1$ if $n=4, b=12$ and $Q \geq 2$ if $n=8, b=12$. This shows that no core player will deviate in the first $T-Q$ periods for the above bounds on $Q$.

Now consider deviations by a periphery player $j$. Note that within a period, the periphery player best responds by linking to the core in the superstar. If a periphery player deviates, she will be punished in all $T-t$ remaining periods. The expected future payoffs in case of deviation are thus $w_{i}(T-t)$. As $\bar{\pi}^{S}>\bar{\pi}^{N}>w_{i}(1) \geq \frac{w_{i}(T-t)}{T-t}$, the expected future payoff of adhering to the strategy $s_{j}^{*}$ is strictly larger than the future payoffs of deviation. Hence, no periphery player will deviate, which shows that the strategy profile $s^{*}$ constitutes a subgame perfect equilibrium. Q.E.D.

Given that in practice rotation schemes are hardly implemented, we now focus on equilibria where the same network structure is played in all periods. We divide the game in two phases: a 'superstar phase' where a superstar with a fixed core is played in the first $T-Q$ periods, and an 'end phase' which consist of the final $Q$ periods.

\footnotetext{
${ }^{27}$ More specifically, if $n=4, b=0$ we have $Q>\frac{2}{3}$ and if $n=8, b=0$ we have $Q>\frac{10}{7}$.
} 
Proposition 2: If we only allow strategies where (1) on the equilibrium path the linking decision of each player does not change and where (2) within each phase the investment decision of each player does not change, then superstars can only be supported in the presence of social benefits.

Proof. The first condition in Proposition 2 implies that all network structures on the equilibrium path should be the same as a superstar's structure, i.e. all networks should be periphery-sponsored stars where a fixed player $i$ is in the core in every period. The second condition in Proposition 2 implies that the end phase should consist of the repeated play of a specific stage-game equilibrium, as both the linking and investment decisions are not allowed to change. In combination, the two conditions result in the repeated play of the Nash star in the final phase, as this is the only stage- game equilibrium that is a periphery-sponsored star. ${ }^{28}$ Hence, in this type of equilibrium, the efficient superstar is played in periods 1 to $T-Q$ and the Nash star is played in the final $Q$ periods. In every period, the same player $i$ fills the core position. Now, consider the following strategy profile $s^{*}$ :

"In each period, the core position is filled by the same player $i$. This player does not form any link, and all other players only form a link to this core player. In the first T-Q periods the core player invests the efficient level $\tilde{y}$ and in the final $Q$ periods the core player invests the stage-game Nash level $\hat{y}$. If some player $j$ deviates in round $t$, play switches to the punishment regime and the deviating player will be punished in rounds $t+1$ to $T$ by the optimal punishment strategy."

First, consider deviations by the core player. As before, the optimal deviation by the core in the superstar phase is to lower her investment to $\hat{y}$, which results in a Nash star. Hence, the core will not deviate in the first $T-Q$ periods if:

$$
(T-Q-t+1) \pi_{C}^{S}+Q \pi_{C}^{N}>\pi_{C}^{N}+(T-t) \frac{w_{i}(T-t)}{T-t},
$$

\footnotetext{
${ }^{28}$ A less stringent requirement would be to impose no additional restrictions on the networks in the final $Q$ rounds, except that the proposed equilibrium should be subgame perfect. In this case, superstars can be sustained for some time in the game without social benefits, but only for the first 36 periods in groups of four and the first 27 periods in groups of eight. Furthermore, the final $Q$ periods would again require changes of the network and involve networks that are not stage-game equilibria. Our aim here is to describe equilibria that avoid such coordination problems.
} 
and will have a profitable deviation if the reverse holds. If $\pi_{C}^{S}<\frac{w_{i}(T-t)}{T-t}$, it is sufficient to consider only period $t=1$, while if $\pi_{C}^{S}>\frac{w_{i}(T-t)}{T-t}$ it is sufficient to consider only the final period of the superstar phase, i.e. $t=T-Q$.

First consider the game without social benefits. As before, $\frac{w_{i}(T-t)}{T-t}=\pi_{C}^{N}$. As $\pi_{C}^{N}>$ $\pi_{C}^{S}$, we consider period $t=1$. Using this in (C.6) reduces the condition to $\pi_{C}^{S}>\pi_{C}^{N}$ which is not true. Thus, the reverse sign holds in (C.6) and a profitable deviation exists for the core player. Hence, the strategy profile $s^{*}$ does not constitute a subgame perfect equilibrium in the absence of social benefits.

Now consider the game with social benefits. With social benefits $\pi_{C}^{S}>w(1) \geq \frac{w_{i}(T-t)}{T-t}$, which implies that it is sufficient to consider the last period of the superstar phase. ${ }^{29}$ Using this in eq. (C.6) yields:

$$
Q>\frac{\pi_{C}^{N}-\pi_{C}^{S}}{\pi_{C}^{N}-w_{i}(Q) / Q}
$$

which provides a condition for the minimal length of the Nash phase, as in condition (C.5). We can substitute and rewrite (C.7) as:

$$
b>\frac{1}{n-1}\left(\frac{(\tilde{y}-\hat{y}) c_{i}-(f(\tilde{y})-f(\hat{y}))}{Q}+\frac{w_{i}(Q)}{Q}-\left(f(\hat{y})-c_{i} \hat{y}\right)\right) .
$$

The right hand side of (C.8) is strictly positive, as $\frac{w_{i}(Q)}{Q} \geq v_{i}=\left(f(\hat{y})-c_{i} \hat{y}\right)$ and $(\tilde{y}-$ $\hat{y}) c_{i}>(f(\tilde{y})-f(\hat{y}))$. In other words, we need positive social benefits to sustain a superstar in this type of equilibrium.

For our game, we can use $\frac{w_{i}(Q)}{Q} \leq w_{i}(1)$ and the fact that the lowest stage-game equilibrium payoff for the treatments with social benefits is obtained in the periphery of the Nash 2-hub. As the payoff in the periphery of the Nash 2-hub is strictly smaller than $\pi_{C}^{S}$, condition (C.7) implies that $Q \geq 1$ in all treatments with social benefits. ${ }^{30}$ In the final $Q$

\footnotetext{
${ }^{29}$ This holds if $(n-1) b>w_{i}(1)+c_{i} \tilde{y}-f(\tilde{y})$, hence when the social benefits or the number of players are sufficiently large. For our experimental game, it holds for all roles in all treatments, except for role D in treatment $n=4, b=12$.

${ }^{30}$ Choosing $Q=T-1$ in (C.8) shows that we need $b>2.09$ if $n=4$ and $b>0.66$ if $n=8$ to sustain a superstar with the low cost player A as the core. If we set $Q=1$, we obtain $b>6.33$ if $n=4$ and $b>4.86$ if $n=8$.
} 
periods, the Nash star will be played, which is a stage-game Nash network. Hence, the core player has no profitable one-stage-deviation.

Finally, consider the periphery players in the game with social benefits. Again, within a period, the periphery players best respond by linking to the core in the superstar. If a periphery player deviates, she will be punished in all $T-t$ remaining periods. The average future payoffs in case of deviation are thus $\frac{w_{i}(T-t)}{T-t}$. As $\pi_{P}^{S}>\pi_{P}^{N} \geq w_{i}(1) \geq \frac{w_{i}(T-t)}{T-t}$, the future payoffs of adhering to the strategy are strictly larger than the future payoffs of deviation. Hence, no periphery player will deviate, which shows that the strategy profile $s^{*}$ constitutes a subgame perfect equilibrium in the game with social benefits, and that superstars can be sustained until period $T-1$. Q.E.D. 